\title{
Immunoglobulin light chain amyloidosis diagnosis and treatment algorithm 2021
}

\author{
M. Hasib Sidiqi ${ }^{1}$ and Morie A. Gertz $\mathbb{B}^{2}$
}

\begin{abstract}
Immunoglobulin light chain amyloidosis (AL) commonly presents with nephrotic range proteinuria, heart failure with preserved ejection fraction, nondiabetic peripheral neuropathy, unexplained hepatomegaly or diarrhea, and should be considered in patients presenting with these symptoms. More importantly, patients being monitored for smoldering multiple myeloma and a monoclonal gammopathy of undetermined significance (MGUS) are at risk for developing AL amyloidosis. MGUS and myeloma patients that have atypical features, including unexplained weight loss; lower extremity edema, early satiety, and dyspnea on exertion should be considered at risk for light chain amyloidosis. Overlooking the diagnosis of light chain amyloidosis leading to therapy delay is common, and it represents an error of diagnostic consideration. Herein we provide a review of established and investigational treatments for patients with AL amyloidosis and provide algorithms for workup and management of these patients.
\end{abstract}

\section{Introduction}

The incidence of AL amyloidosis is estimated to be three to five patients per million per year ${ }^{1}$. This statistic would make it approximately one-fifth as common as multiple myeloma ${ }^{2}$. In the United Kingdom, the incidence is $\sim 1$ per $100,000^{3}$. The Medicare claims database suggests that the mean age of AL amyloidosis at diagnosis is 63 with an incidence of 10-14 patients per million per year with a prevalence higher in males ${ }^{4}$. Wild-type transthyretin (TTR) amyloidosis is increasingly being recognized and may be present in a quarter of the elderly at postmortem and is seen in 13-19\% of patients with heart failure and preserved ejection fraction, likely making it the most common form of systemic amyloidosis ${ }^{5}$.

The diagnosis of AL amyloidosis should be considered by physicians in any patient seen with nephrotic range proteinuria, heart failure with preserved ejection fraction ${ }^{6}$, nondiabetic peripheral neuropathy ${ }^{7}$, unexplained hepatomegaly ${ }^{8}$, or diarrhea. However these signs and symptoms are not specific to amyloidosis. Heart failure with

Correspondence: Morie A. Gertz (gertz.morie@mayo.edu)

${ }^{1}$ Haematology Department, Fiona Stanley Hospital, Perth, WA, Australia

${ }^{2}$ Division of Hematology, Department of Internal Medicine, Mayo Clinic,

Rochester, MN, USA preserved ejection fraction, one of the most common manifestations of $\mathrm{AL}$ amyloidosis, can be misdiagnosed because the echocardiogram has nonspecific findings. Wall thickening can be misinterpreted as hypertension with hypertrophy or hypertrophic cardiomyopathy ${ }^{9}$. Cardiac magnetic resonance imaging with gadolinium is a more specific test, however is not a screening test and often only ordered if amyloidosis is suspected ${ }^{10}$. A pseudoinfarction pattern seen on the EKG could be misinterpreted as true ischemic disease. Patients with peripheral neuropathy and a monoclonal gammopathy are frequently misdiagnosed as CIDP (chronic inflammatory demyelinating polyneuropathy $)^{11}$. Patients often undergo unnecessary therapies such as immunoglobulin infusions and plasma exchange for many months prior to evaluation for AL amyloidosis. Other clinical features of AL amyloidosis such as tongue enlargement or periorbital purpura, whilst highly specific, are only found in $15 \%$ of patients and are not effective screening tools for $\mathrm{AL}$ amyloidosis.

For the cancer provider following patients with monoclonal gammopathy of undetermined significance (MGUS) or smoldering multiple myeloma, it is important to keep in mind that these patients are not monitored 
solely for the development of myeloma. Some develop lymphoma or Waldenström macroglobulinemia, and a small percentage develop light chain amyloidosis ${ }^{12}$. At Mayo Clinic, 9\% of all patients seen with a monoclonal gammopathy are ultimately proven to have light chain amyloidosis. Even adjusting for referral bias, $3-4 \%$ of all patients with monoclonal proteins have light chain amyloidosis. Furthermore, if a provider does not see one patient with AL amyloidosis for every five patients with multiple myeloma, it is likely the diagnosis is being overlooked $^{13}$.

The ongoing rates of early mortality in newly diagnosed AL amyloidosis suggest there continue to be significant delays in diagnosis ${ }^{14}$. Nearly $20 \%$ of patients succumb to the disease within 6 months of diagnosis, and this statistic has shown no improvement in 40 years, suggesting, that patients who are diagnosed at an advanced state cannot be helped despite major advances in therapy for this disease.

An online survey from the Amyloid Research Consortium indicates that $37 \%$ of patients are diagnosed over 1 year from the onset of initial symptoms with a median of three physician visits before a diagnosis is established. Cancer care providers constitute $34 \%$ of the specialists that are diagnosing this disorder, far greater than nephrologists, cardiologists, and gastroenterologists ${ }^{15}$.

If $\mathrm{AL}$ amyloidosis is suspected, particularly in patients who have multi-organ dysfunction, biopsies are not the first step in screening. Currently, $71 \%$ of patients that are seen have cardiac involvement, $58 \%$ have renal involvement, $23 \%$ have nerve involvement, and $16 \%$ have liver involvement. Despite these numbers, the majority of patients with cardiac, renal, hepatic, and nerve problems will not have AL amyloidosis. The first screening test for these patients, as shown in the algorithm (Fig. 1), would be serum immunofixation and an immunoglobulin free light chain assay for $\mathrm{k}$ and $\lambda$ immunoglobulin light chains ${ }^{16}$. If the patient has cardiac dysfunction, a pyrophosphate scan, which should be available in most hospitals in the developed world, should also be performed ${ }^{17}$. If these screening tests are positive, the diagnostic pathway is clear. A simple subcutaneous fat aspirate (https://www.youtube.com/ watch? $\mathrm{v}=$ tctYTmxd9gQ) and a bone marrow biopsy will demonstrate amyloid deposits in over $85 \%$ of patients with immunoglobulin light chain abnormalities and confirm a diagnosis of AL amyloidosis. If the bone marrow biopsy and fat aspirate are negative and only if the index of suspicion is high would the patient next move to a direct organ biopsy of the heart, liver, kidney, nerve, etc. Patients with echocardiographic evidence suggesting cardiac amyloidosis, usually demonstrate thickened ventricular walls, Doppler evidence of poor diastolic filling and abnormal longitudinal strain. These patients need to undergo a pyrophosphate scan. A positive scan in the absence of a monoclonal protein in the serum and urine is sufficient for the diagnosis of cardiac TTR amyloidosis ${ }^{18}$. These patients should be referred to a cardiologist for further investigation and management. However distinguishing TTR amyloidosis and $\mathrm{AL}$ amyloidosis can be difficult given the increased prevalence of MGUS in patients with TTR amyloidosis and the rare phenomena of two types of amyloidosis presenting concurrently, making subtyping of the amyloid deposits critical $^{19-22}$.

\section{Classification of amyloid}

Accurate typing of the protein subunit responsible for amyloid deposition is important since it directs treatment. This can range from observation to intensive treatment with chemotherapy as is in the cases in systemic AL amyloidosis, making subtyping critical for appropriate management. Historically, immunohistochemistry ${ }^{23,24}$ and immunofluorescence ${ }^{25}$ techniques have been used to subtype the amyloid deposits. However these techniques have limitations. In light chain amyloidosis, commercial antisera purchased to detect $\kappa$ and $\lambda$ immunoglobulin light chains are usually directed against epitopes on the constant region of the immunoglobulin light chain. When amyloid light chains are deposited in tissues, usually only a fragment of the intact light chain is deposited, typically the variable portion $\left(\mathrm{V}_{\mathrm{L}}\right)$ with a molecular weight of $\sim 12 \mathrm{kd}$. A normal intact light chain has a molecular weight of $25 \mathrm{kd}$, suggesting there has been deletion of the constant portion of the light chain, making the immunoglobulin fragment unrecognizable to commercial antisera. Secondly, the light chains in amyloidosis are known to misfold, and the potential of a previously exposed epitope no longer being accessible to the commercial antibody exists, rendering it unidentifiable ${ }^{26}$. Finally, there are at least 30 different types of amyloid proteins, and few centers are equipped with such a large panel of antisera, making it next to impossible to identify rare forms of amyloidosis, such as fibrinogen, LECT2, apolipoprotein, and lysozyme.

The gold standard for typing is laser capture mass spectroscopic proteome analysis ${ }^{27}$. Amyloid deposits are directly micro dissected from a glass slide, and the technique can be performed on archived paraffin-embedded tissues. Peptides are sequenced by a mass spectrometer and then compared with libraries of proteins for identification $^{28,29}$. Although expensive and not available in all laboratories, proteomic analysis with mass spectroscopy remains the gold standard for identification of the amyloid protein subunit.

In a review of over 4000 proteomic analyses of amyloid deposits, $62 \%$ were of immunoglobulin origin. However, a full $38 \%$ were not of immunoglobulin origin, and chemotherapy would have been contraindicated. Nonimmunoglobulin forms of amyloidosis included AA 


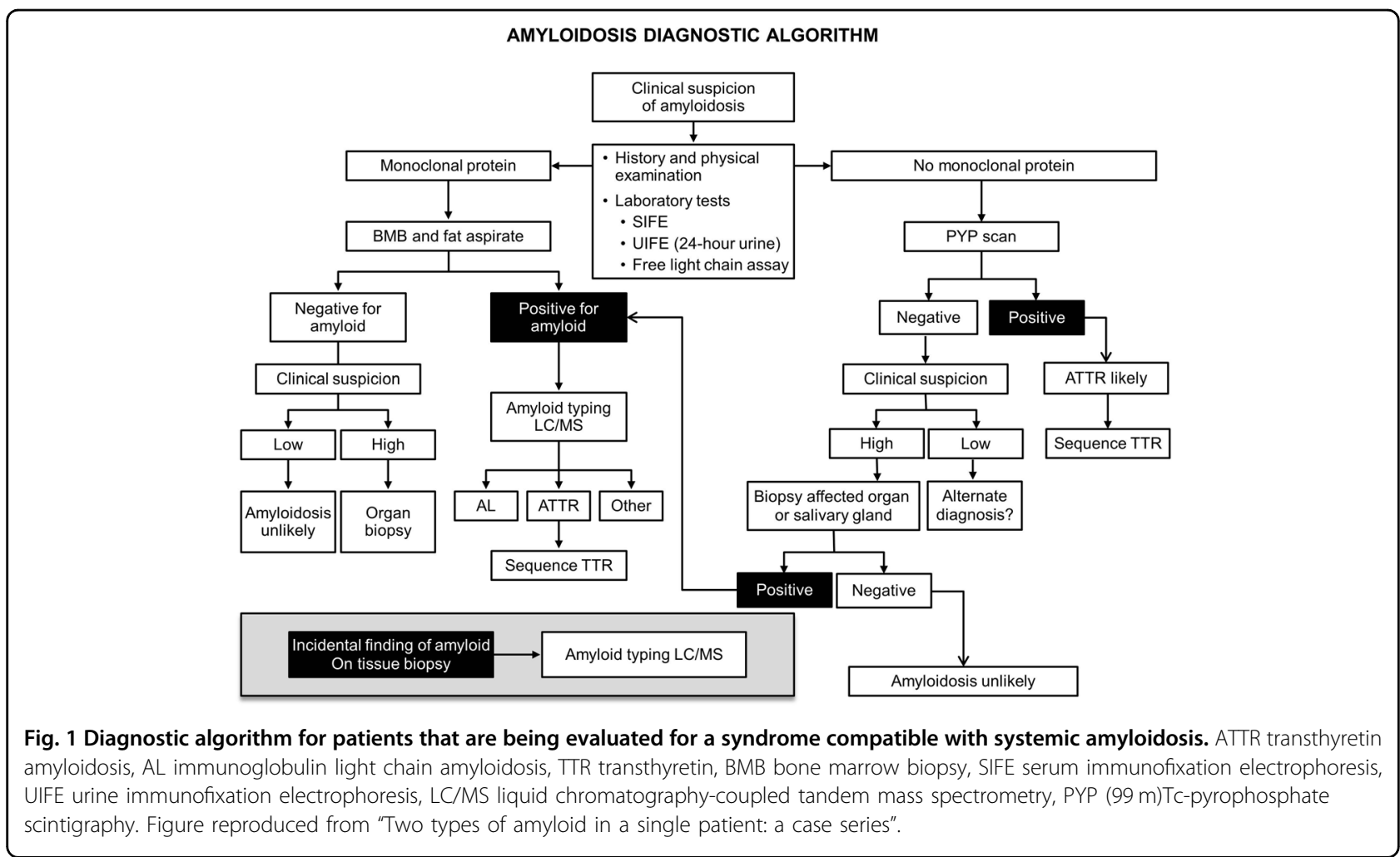

amyloidosis, ALECT2, A-insulin, A-fibrinogen, and Agelsolin (Kurtin P. personal communication) ${ }^{30-33}$.

\section{Patient is referred from a specialist with biopsy-proven amyloid}

Many specialists, when encountering a patient with biopsy of an organ containing amyloid, refer to a cancer care provider uncertain of the type of amyloidosis. The first step for all biopsied tissues, would be mass spectroscopic analysis (Fig. 1). In patients with AL amyloidosis, measurement of bone marrow plasma cells ${ }^{34}$ and FISH genetics $^{35}$, as would be done in multiple myeloma patients, are indicated. To accurately stage AL amyloidosis testing for NT-proBNP, troponin, and the difference between the involved and uninvolved immunoglobulin free light chain is needed ${ }^{16}$. Cardiac imaging either with echocardiography or magnetic resonance imaging can further rdelineate degree of cardiac involvement, critical for prognosis ${ }^{36}$. For patients with light chain amyloidosis in the absence of symptoms, the role of routine skeletal imaging, as is done in multiple myeloma, is not well defined due to a lack of high-quality evidence.

If TTR amyloid is identified by mass spectroscopic analysis, clinically these patients should have presented with peripheral neuropathy or cardiomyopathy. For patients with cardiac symptoms a pyrophosphate scan of the heart should be performed and if strongly positive would suggest that the amyloid is of TTR origin ${ }^{37}$. Any patient with TTR amyloidosis should have gene sequencing of the TTR gene to distinguish wild-type TTR, as is seen in senile cardiac amyloidosis, from the very rare mutations of TTR that lead to inherited amyloidosis ${ }^{38}$. The genetic mutations causing TTR amyloidosis vary among ethnicities with the most common V30M mutation having clusters in Portugal, Japan and Sweden, whilst others are less common such as the T60A mutation seen in Ireland. Since familial amyloidosis is not treated with chemotherapy, these patients should be referred for genetic counseling, consideration of liver transplant, diflunisal ${ }^{39,40}$ or doxycycline therapy ${ }^{41}$, or one of the agents that suppress translation of liver TTR messenger $\mathrm{RNA}^{42,43}$ into the fully-formed TTR protein. Patients with wild-type TTR amyloidosis are usually over the age of $70,90 \%$ are men, and half have carpal tunnel syndrome ${ }^{44}$. Tafamidis, an agent that stabilizes the TTR proteins, is approved therapy for TTR cardiac amyloidosis.

Staging of AL amyloidosis is based on a four-point system where one point is assigned for a DFLC $>18 \mathrm{mg} /$ $\mathrm{dL}$, a cardiac troponin $T>0.025 \mathrm{mcg} / \mathrm{L}$, or an NT-proBNP $\geq 1800 \mathrm{ng} / \mathrm{L}$. This provides a staging system of I, II, III, and IV based on the number of points assigned $(0,1,2$, or 3$)$. The staging system has been validated in multiple datasets, including patients treated with stem cell transplantation, patients on clinical trials, and non-transplant patients treated with standard chemotherapy ${ }^{45}$. Other effective staging systems include a European staging 
Current Treatment Algorithm for Light Chain Amyloidosis

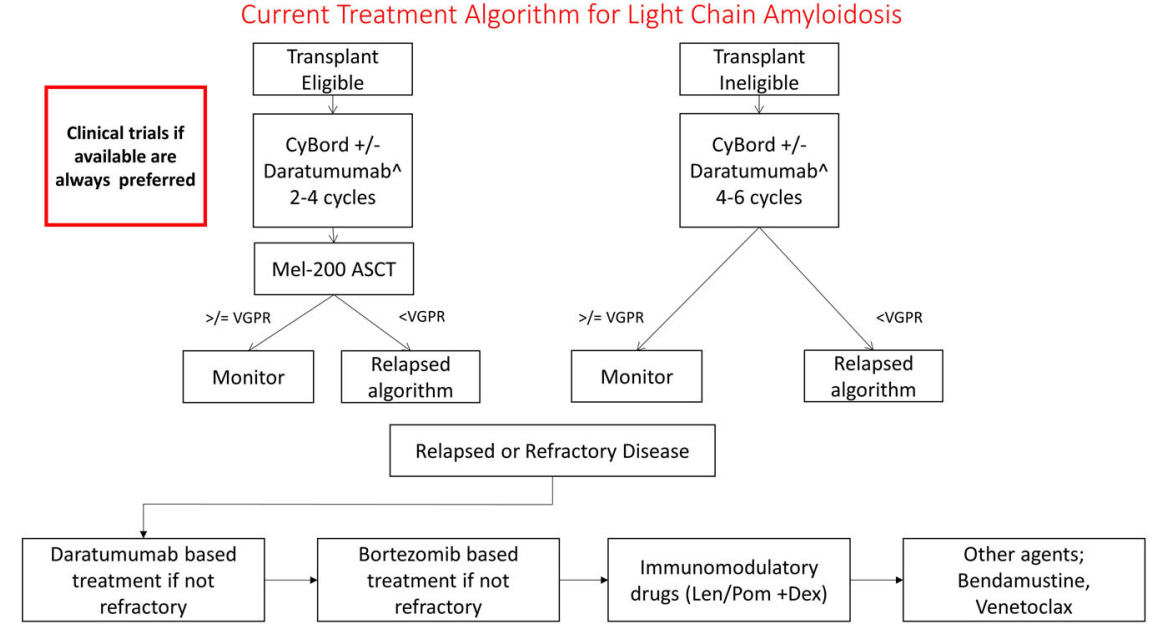

Fig. 2 Current treatment algorithm in light chain amyloidosis. CybBord cyclophosphamide, bortezomib, and dexamethasone, ASCT autologous stem cell transplant, VGPR very good partial response, Len lenalidomide, Pom pomalidomide, Dex dexamethasone. ^Where available induction with daratumumab plus CyBord is recommended. If unavailable CyBord is induction is recommended. *Daratumumab base treatment is preferred at first relapse if patient is previously unexposed to this agent.

system where Mayo 2004 stage 3 was subclassified into three substages using systolic blood pressure and NTproBNP at $100 \mathrm{mmHg}^{46}$ and $8500 \mathrm{ng} / \mathrm{mL}^{47}$, respectively.

\section{Therapy of amyloidosis}

The first successful treatment for AL amyloidosis was melphalan and prednisone introduced in $1972^{48}$. Subsequently autologous stem cell transplantation was reported in $1996^{49}$, and high-dose dexamethasone in $1997^{50}$. Melphalan and dexamethasone combination therapy was reported in $2004^{51}$. With the introduction of novel agents for treatment of myeloma, many of these were subsequently utilized in AL amyloidosis including immunomodulatory drugs (IMIDs) such as thalidomide ${ }^{52,53}$, lenalidomide ${ }^{54,55}$, and pomalidomide ${ }^{56,57}$, as well as combinations of IMIDs with alkylating agents ${ }^{58}$. However IMIDs are poorly tolerated in patients, particularly those with cardiac AL amyloidosis ${ }^{59,60}$. The first step in assessing therapy for a patient with AL amyloidosis, as shown in our algorithm (Fig. 2), is determination of their eligibility for stem cell transplantation. Using transplantation in $\mathrm{AL}$ amyloidosis is theoretically better than it is for multiple myeloma. Unlike multiple myeloma, the tumor mass being treated is less with a median of $\sim 10 \%$ plasma cells at diagnosis and a median dFLC of only $24 \mathrm{mg} / \mathrm{dL}$. Unfavorable genetics, seen in nearly a quarter of patients with multiple myeloma [such as $1 \mathrm{q}+, \mathrm{t}(4 ; 14)$, and $-17 \mathrm{p}$ ] are present in $<5 \%$ of patients with light chain amyloidosis. The proliferative rate of plasma cells is lower in AL amyloidosis patients, suggesting that once a response is obtained, it is likely to be more durable than is seen in multiple myeloma ${ }^{61}$. In fact, in the pre-novel agent era, 10year survival of patients with AL amyloidosis undergoing stem cell transplantation was $43 \%{ }^{62}$. A prospective randomized trial of melphalan and dexamethasone with stem cell transplant also favored stem cell transplantation, although the comparator arm did not contain novel agents $^{63}$. With careful patient selection, the therapy-related mortality has been reduced to $\sim 2 \%{ }^{64,65}$. Patients that do not achieve greater than a VGPR can have bortezomibbased consolidation posttransplant, which significantly upgrades treatment response posttransplant ${ }^{66}$. A prospective randomized trial demonstrated an improved survival outcome with bortezomib-dexamethasone prior to stem cell transplant ${ }^{67}$. Our current approach is to manage all transplant eligible patients with induction therapy followed by stem cell collection and transplant (Fig. 2).

However it is important to note that no more than $25 \%$ of newly diagnosed patients are eligible by virtue of age, renal function, and extent of cardiac failure. The remaining $75-80 \%$ are candidates for chemotherapy. Melphalan and dexamethasone demonstrates impressive survival in patients that are capable of receiving full-dose therapy with a median survival of just $<8$ years ${ }^{68}$. There have been reports of cyclophosphamide-thalidomide-dexamethasone ${ }^{69}$, lenalidomide-dexamethasone, melphalan-dexamethasonelenalidomide, cyclophosphamide-lenalidomidedexamethasone $\mathrm{e}^{70}$, but none of these are currently used in the Mayo Clinic algorithm due to toxicity and the preference for bortezomib. It should be noted that lenalidomide raises the NT-proBNP in AL patients ${ }^{71}$. CyBorD or VCD (cyclophosphamide-bortezomib-dexamethasone) was first reported to be effective in 2012. In the original iteration, cyclophosphamide was given orally weekly, dexamethasone orally weekly, and bortezomib subcutaneously weekly. In this original trial, 17 patients were treated, 10 with 
symptomatic cardiac involvement with a $94 \%$ response rate, and $71 \%$ complete response rate with an additional three patients who were previously deemed ineligible for stem cell transplant to become eligible ${ }^{72}$. These results were validated in over 230 patients with AL amyloidosis, demonstrating a median survival in excess of six years, with all patients surviving in stage 1 disease and a median survival of $<1$ year in stage 4 disease $^{47}$. Survival was dependent on response depth, with patients achieving a VGPR or better having the best outcome. Achievement of a VGPR is used in the algorithm to determine whether second-line therapy should be considered. The utility of frontline bortezomib therapy in $\mathrm{AL}$ amyloidosis is further highlighted by a phase III randomized open-label trial comparing bortezomib, melphalan, and dexamethasone with melphalan and dexamethasone in newly diagnosed patients. Response rates were higher in the bortezomib containing arm (79 vs. $52 \%$ ) as was the rate of VGPR or CR (64 vs. $395 \%)^{73}$. Overall survival was improved in the bortezomib arm with a two fold reduction in mortality rate. The UK national Amyloidosis center reported a prospective observational study of 915 patients treated with frontline bortezomib-based therapy for AL amyloidosis showing an overall response rate (ORR) of $65 \%$ with $49 \%$ of patients achieving a CR or $\mathrm{VGPR}^{74}$. Median overall survival was 72 months and not reached in those achieving a stringent $\mathrm{CR}(\mathrm{dflc}<10 \mathrm{mg} / \mathrm{L})$. In using bortezomib-based therapy, one needs to be aware that response rate is lower in patients with $\mathrm{t}(11 ; 14)^{75}$, a genetic abnormality seen in nearly $50 \%$ of patients with AL amyloidosis. The presence of $t$ $(11 ; 14)$ should lead one to strongly consider stem cell transplantation over bortezomib, since this genetic abnormality does not have an unfavorable impact in transplanted patients. Predictors of early death after therapy initiation include the Mayo stage and greater than two organs involved. The value of addition of cyclophosphamide to bortezomib is debatable with some data suggesting limited additional benefit ${ }^{76}$.

\section{Daratumumab}

Daratumumab, a monoclonal antibody to CD38, approved for the treatment of relapsed multiple myeloma as a single agent as well as in combination with lenalidomide or bortezomib, clearly shows activity in the treatment of patients with $\mathrm{AL}$ amyloidosis ${ }^{77}$ and appears to have a low-toxicity profile. Early reports suggested a high response rate $(76 \%)$ in heavily pretreated patients ${ }^{78}$. These data were confirmed by a number of other retrospective studies $^{79-81}$. Two prospective phase II studies of daratumumab in combination with dexamethasone for relapsed AL amyloidosis have been with encouraging results. A multicenter French study of 40 patients receiving 6 months of daratumumab therapy showed an ORR of $70 \%$ with a median time to response of 1 week $^{82}$. Similarly a phase II study conducted by the Boston group showed that daratumumab and dexamethasone (planned therapy for 2 years) led to a high ORR of $90 \%$ with a CR/VGPR rate of $86 \%$ and median time to response of 4 weeks ${ }^{83}$. The rates of organ response were 31 and $67 \%$ for renal response and 29 and 50\% for cardiac response in the French and Boston studies respectively. Similar rates of organ response were reported, $52 \%$ renal and $55 \%$ cardiac, in a retrospective study of 72 patients receiving daratumumab and dexamethasone conducted at Stanford University ${ }^{84}$. Kimmich et al. reported 168 consecutive patients with relapsed AL amyloidosis treated with daratumumab, 62 of whom received this in combination with bortezomib and dexamethasone showing that this combination was well tolerated and efficacious in this population ${ }^{85}$. The early reports of success of daratumumab in AL amyloidosis led to the phase III ANDROMEDA study of VCd with or without daratumumab as upfront therapy for newly diagnosed AL amyloidosis patients. Early data reported from the safety run that included 28 patients showed an ORR of $96 \%$ and a CR rate of $54 \%^{86}$. The study reported interim analysis showing a CR rate of $53 \%$ for Dara- $\mathrm{VCd}$ and $18 \%$ for VCd (odds ratio, 5.1; 95\% CI, 3.2-8.2; $P<0.0001$ ) and better organ responses with the daratumumab containing regimen, cardiac response rate was $42 \%$ for Dara- $\mathrm{VCd}$ and $22 \%$ for VCd $(P=0.0029)$; 6-month-renal response rate was $54 \%$ and $27 \%$, respectively $(P<0.0001)^{87}$. It was based on these impressive data that the FDA granted accelerated approval in January of 2021 for daratumumab in combination with bortezomib, cyclophosphamide and dexamethasone for newly diagnosed AL amyloidosis. This is the first FDA approval of an agent specifically for the treatment of AL amyloidosis and represents a landmark moment, suggesting that daratumumab will be a mainstay of treatment for $\mathrm{AL}$ amyloidosis in the future. Isatuximab is another monoclonal antibody to CD38 that has shown efficacy in myeloma and is now being studied in the treatment of AL amyloidosis. The SWOG S1702 study is a phase II clinical trial assessing the efficacy of Isatuximab in patients with previously treated $\mathrm{AL}$ amyloidosis (NCT03499808). Preliminary data on 36 patients recruited to the study were presented at the 62nd American Society of Hematology meeting showing an ORR of $77 \%$ and a median time to response of 1.1 months.

\section{Other agents}

Because of the high prevalence of $t(11 ; 14)$ in AL amyloidosis patients, Venetoclax ${ }^{88}$, which has activity in multiple myeloma, particularly in those with the $t(11 ; 14)$, would be a natural candidate for the treatment of $\mathrm{AL}$ amyloidosis. A number of case reports showed efficacy in patients with AL amyloidosis ${ }^{89-92}$. A case series of 12 patients treated at Mayo Clinic showed hematologic response in 7 out of 8 evaluable patients, ORR $88 \%{ }^{93}$. However given concerns regarding infection and mortality 
when used in combination with bortezomib and dexamethasone for myeloma in the phase III BELLINI study, use of this agent in AL amyloidosis patients who often have organ dysfunction needs to be carefully considered. A proposed phase I clinical trial is currently suspended (NCT03000660).

Carfilzomib, the second-generation proteasome inhibitor, has been tested ${ }^{94}$. A high incidence of cardiac involvement with AL amyloid makes it a challenging agent to use. Traditional pre- and post-hydration can aggravate patients predisposed to congestive heart failure. Carfilzomib is associated with cardiotoxicity in nearly $10 \%$ of patients. A review of Medicare admissions showed that carfilzomibtreated patients had a higher risk of hospitalization ${ }^{95}$. Hematologic responses have been reported in a small cohort of AL amyloidosis patients treated with upfront carfilzomib based therapy ${ }^{96}$. However its potential cardiotoxicity may be a barrier for wider implementation of this agent. Ixazomib has been shown to have a manageable toxicity profile and efficacious in patients with AL amyloidosis $^{97,98}$. A phase 3 trial of ixazomib-dexamethasone versus physician-selected standard of care (TOURMALINE-AL1) was discontinued after it failed to show a significant improvement in hematologic response over physician choice (NCT01864018).

Bendamustine is an agent that is commonly used in non-Hodgkin lymphoma and has shown efficacy in myeloma. In a retrospective study of 122 patients with $\mathrm{AL}$ amyloidosis the combination of bendamustine with oral prednisolone led to an ORR of 35\% with response rates higher in patients with IgM AL amyloidosis (58 vs. $28 \%$ ). The toxicity profile was manageable and consistent with the use of bendamustine in other hematological malignancies. A prospective phase II study examined efficacy of bendamustine in combination with dexamethasone for relapsed AL amyloidosis and showed an ORR of $57 \%$ and organ response rate of $29 \%{ }^{99}$. Grade 3-4 adverse events were seen in $65 \%$ and most commonly included myelosuppression, fatigue, and nausea.

Doxycycline has been used in patients with both $\mathrm{AL}^{100}$ and $\mathrm{TTR}^{101-103}$ amyloidosis with cardiac involvement. In vitro, doxycycline appears to disaggregate formed fibrils ${ }^{104}$. A trial from Mayo Clinic demonstrated that patients who achieved a hematologic response to stem cell transplant had a significantly longer overall survival post stem cell transplantation when given doxycycline compared to those receiving penicillin ${ }^{105}$. In a second study, which was case control, 26 patients receiving doxycycline were matched to 50 controls. The response rate was significantly higher in the doxycycline compared to controls, and the 12 -month survival was 84 vs. $58 \%$. Although there is no high-quality evidence and it has not been validated in a prospective randomized trial, doxycycline is a consideration if no other therapies are feasible. Preclinical studies suggest it may modulate amyloidogenic light chain mediated proteotoxicity in cardiac myocytes and studies using adjuvant doxycycline as part of therapy for AL amyloidosis have shown that this agent can be safely added to current treatment regimens ${ }^{106,107}$.

\section{Monoclonal antibodies to dissolve amyloid}

Although chemotherapy can effectively reduce the light chain burden and disrupt further deposition in AL amyloidosis, it does nothing for resident amyloid in tissues. Although a number of agents in this setting have been studied over the recent years, only one is currently being evalusted in later stage clinical trials. The murine monoclonal antibody, 11-1F4, recognizes an amyloid-associated conformational epitope ${ }^{108}$. In a phase Ia/Ib study of 27 patients, 24 were evaluable for organ response and 12 of 18 (67\%) patients with renal and/or cardiac involvement achieved a response. No toxicity >grade 3 was recognized $^{109-111}$. This agent is being studied further in phase II and III clinical trials. Amyloid fibril targeted therapy with monoclonal antibodies is promising for the management of all forms of amyloidosis. Dissolution of amyloid fibrils may improve organ function.

\section{Organ transplantation}

In AL amyloidosis, selected patients may successfully undergo renal or cardiac transplantation to assist with organ recovery. For patients that have single-organ involvement and control of the plasma cell proliferative process, organ transplantation may be considered. Stem cell transplantation can be safely performed in patients with dialysis-dependent ${ }^{112}$ renal failure ${ }^{113}$. Failure to achieve a complete response is no longer considered a contraindication to organ transplantation because of the increased availability of therapeutic options and direct organ donor programs. Once the patient has an established complete response, consideration of renal transplantation may be undertaken. Cardiac transplantation has also been performed in patients with $\mathrm{AL}$ amyloidosis ${ }^{114}$. Patients with advanced cardiac disease are unlikely to tolerate therapy well and achieve deep responses prior to consideration of organ transplantation. There have been cases of patients receiving cardiac allografting first, followed by chemotherapy or autologous stem cell transplantation to achieve remission of the AL amyloidosis ${ }^{115-117}$. Long-term survivorship has been reported in highly selected patients who fulfill the criteria of deep hematologic response and single-organ involvement ${ }^{118}$. Lenalidomide therapy is best avoided in organ transplant recipients that are considered for post-organ transplant chemotherapy ${ }^{119}$.

\section{Conclusion}

Physician alertness and suspicion to the possibility of amyloidosis is a critical first step in diagnosing 
AL amyloidosis. This would allow earlier diagnosis, with less organ dysfunction, a setting in which treatments are likely to be most efficacious. Those diagnosed with advanced cardiac disease are unlikely to benefit from therapy and have a high mortality in the first few months of diagnosis. Once AL amyloidosis is suspected, the diagnosis can usually be made noninvasively, and organ biopsy is not generally required. Mass spectroscopic analysis should be standard for all newly diagnosed patients with amyloidosis to ensure correct classification of the protein subunit, and where unavailable immunohistochemical techniques may be utilized. All patients with light chain amyloidosis need cardiac biomarkers, free light chain measurements, and a bone marrow, with a thorough cardiac evaluation. The treatment of choice remains chemotherapy directed at the plasma cell clone producing the amyloidogenic light chain. In select cases stem cell transplantation may be considered, however only a minority of patients are eligible for this treatment and with the availability of newer agents that achieve deep responses, the utility of stem cell transplantation is likely to be questioned moving forward. For patients that are not transplant eligible but able to tolerate therapy, the standard of care would be combination therapy with daratumumab, bortezomib, cyclophosphamide, and dexamethasone based on the recent FDA approval of this combination for AL amyloidosis. Where daratumumab is unavailable, bortezomib-based regimens are preferred. For very frail patients, all oral therapy with melphalan and dexamethasone is appropriate. Second-line therapy can include immunomodulatory-based therapies, and other agents used in multiple myeloma, although data with use of these agents in this setting are sparse. If daratumumab has not been used upfront, we recommend using this agent at relapse with data showing very high response rates in this setting. Anti-amyloid antibodies are likely to have a potential role in the future management of these patients.

\section{Conflict of interest}

M.H.S. reports honoraria from Amgen, Bristol Myers Squibb, and Celgene. M.A.G. reports personal fees from lonis, personal fees from Alnylym, personal fees from Prothena, personal fees from Celgene, personal fees from Janssen, grants and personal fees from Spectrum, personal fees from Annexon, personal fees from Appellis, personal fees from Amgen, personal fees from Medscape, personal fees from Physicians Education Resource, personal fees from Abbvie, personal fees from Research to Practice, from Teva, outside the submitted work.

\section{Publisher's note}

Springer Nature remains neutral with regard to jurisdictional claims in published maps and institutional affiliations.

Received: 30 January 2021 Revised: 11 April 2021 Accepted: 28 April 2021 Published online: 15 May 2021

\section{References}

1. Kyle, R. A. et al. Incidence and natural history of primary systemic amyloidosis in Olmsted County, Minnesota, 1950 through 1989. Blood 79, 1817-1822 (1992).

2. Costa, L. J. et al. Recent trends in multiple myeloma incidence and survival by age, race, and ethnicity in the United States. Blood Adv. 1, 282-287 (2017).

3. Pinney, J. H. et al. Systemic amyloidosis in England: an epidemiological study. Br J Haematol. 161, 525-532 (2013).

4. Quock, T. P., Yan, J. T., Chang, E., Guthrie, S. D. \& Broder, M. S. Epidemiology of $\mathrm{AL}$ amyloidosis in a US commercially insured population. Blood. 130, 5335-5335 (2017).

5. Tanskanen, M. et al. Senile systemic amyloidosis affects $25 \%$ of the very aged and associates with genetic variation in alpha2-macroglobulin and tau: a population-based autopsy study. Ann. Med. 40, 232-239 (2008).

6. Schelbert, E. B. \& Miller, C. A. Cardiac amyloidosis as a potential confounder in heart failure with preserved ejection fraction trials. JACC Heart Fail. 5, 617 (2017).

7. Mauermann, M. L. Paraproteinemic neuropathies. Continuum (Minneap Minn). 20 (5 Peripheral Nervous System Disorders), 1307-1322 (2014).

8. Ye, L., Shi, H., Wu, H. M. \& Wang, F. Y. Primarily isolated hepatic involvement of amyloidosis: a case report and overview. Medicine (Baltimore) 95, e5645 (2016).

9. Cacciapuoti, $F$. The role of echocardiography in the non-invasive diagnosis of cardiac amyloidosis. J. Echocardiogr. 13, 84-89 (2015).

10. Patel, A. R. \& Kramer, C. M. Role of cardiac magnetic resonance in the diagnosis and prognosis of nonischemic cardiomyopathy. JACC CardiovasC Imaging. 10, 1180-1193 (2017).

11. Graus, F. \& Dalmau, J. Paraneoplastic neuropathies. Curr. Opin. Neurol. 26, 489-495 (2013).

12. Rosenbaum, E., Marks, D. \& Raza, S. Diagnosis and management of neuropathies associated with plasma cell dyscrasias. Hematol. Oncol. 36, 3-14 (2018).

13. McCausland, K. L. et al. Light chain (AL) amyloidosis: the journey to diagnosis. Patient 11, 207-216 (2018).

14. Muchtar, E. et al. Improved outcomes for newly diagnosed AL amyloidosis between 2000 and 2014: cracking the glass ceiling of early death. Blood 129, 2111-2119 (2017).

15. Lousada, I., Comenzo, R. L., Landau, H., Guthrie, S. \& Merlini, G. Light chain amyloidosis: patient experience survey from the amyloidosis research consortium. Adv. Ther. 32, 920-928 (2015).

16. Palladini, G. et al. Circulating free light chain measurement in the diagnosis, prognostic assessment and evaluation of response of $\mathrm{AL}$ amyloidosis: comparison of Freelite and N latex FLC assays. Clin. Chem. Lab. Med. 55, 1734-1743 (2017).

17. Ikram, A. Bone scintigraphy for early detection of transthyretin cardiac amyloidosis. J. Pak. Med. Assoc. 66, 1045 (2016).

18. Gillmore, J. D. et al. Nonbiopsy diagnosis of cardiac transthyretin amyloidosis. Circulation 133, 2404-2412 (2016)

19. Papa, R. et al. Two types of systemic amyloidosis in a single patient. Amyloid 27, 275-276 (2020)

20. Sidiqi, M. H. et al. Two types of amyloidosis presenting in a single patient: a case series. Blood Cancer J. 9, 30 (2019).

21. Phull, $\mathrm{P}$. et al. Monoclonal gammopathy of undetermined significance in systemic transthyretin amyloidosis (ATTR). Amyloid 25, 62-67 (2018).

22. Sidiqi, M. H. et al. Monoclonal gammopathy plus positive amyloid biopsy does not always equal AL amyloidosis. Am. J. Hematol. 94, E141-E143 (2019).

23. Menter, T., Bachmann, M., Grieshaber, S. \& Tzankov, A. A more accurate approach to amyloid detection and subtyping: combining in situ Congo red staining and Immunohistochemistry. Pathobiology 84, 49-55 (2017).

24. Picken, M. M. \& Westermark, P. Amyloid detection and typing: summary of current practice and recommendations of the consensus group. Amyloid 18, 48-50 (2011).

25. Collins, A. B., Smith, R. N. \& Stone, J. R. Classification of amyloid deposits in diagnostic cardiac specimens by immunofluorescence. Cardiovasc. Pathol. 18, 205-216 (2009).

26. Rocken, C., Schwotzer, E. B., Linke, R. P. \& Saeger, W. The classification of amyloid deposits in clinicopathological practice. Histopathology 29, 325-335 (1996).

27. Winter, M., Tholey, A., Kristen, A. \& Röcken, C. MALDI mass spectrometry imaging: a novel tool for the identification and classification of amyloidosis. Proteomics 17, 1700236 (2017). https://doi.org/10.1002/pmic.201700236. 
28. Brambilla, F. et al. Reliable typing of systemic amyloidoses through proteomic analysis of subcutaneous adipose tissue. Blood 119, 1844-1847 (2012).

29. Maleszewski, J. J. et al. Relationship between monoclonal gammopathy and cardiac amyloid type. Cardiovasc. Pathol. 22, 189-194 (2013).

30. Geller, H. I. et al. Prevalence of monoclonal gammopathy in wild-type transthyretin amyloidosis. Mayo Clin. Proc. 92, 1800-1805 (2017).

31. Sidiqi, M. H. et al. Autologous stem cell transplantation in patients with AL amyloidosis with impaired renal function. Bone Marrow Transplant. 54 1775-1779 (2019).

32. Aljama, M. A. et al. Comparison of different techniques to identify cardiac involvement in immunoglobulin light chain (AL) amyloidosis. Blood Adv. $\mathbf{3}$ 1226-1229 (2019).

33. Kourelis, T. V. et al. Presentation and outcomes of localized immunoglobulin light chain amyloidosis: the Mayo Clinic Experience. Mayo Clin. Proc. 92 908-917 (2017).

34. Kourelis, T. V. et al. Coexistent multiple myeloma or increased bone marrow plasma cells define equally high-risk populations in patients with immunoglobulin light chain amyloidosis. J. Clin. Oncol. 31, 4319-4324 (2013).

35. Gertz, M. A., Dispenzieri, A. \& Muchtar, E. Importance of FISH genetics in light chain amyloidosis. Oncotarget. 8, 81735-81736 (2017).

36. Grogan, M., Dispenzieri, A. \& Gertz, M. A. Light-chain cardiac amyloidosis: strategies to promote early diagnosis and cardiac response. Heart 103 1065-1072 (2017).

37. Papantoniou, V. et al. Imaging of cardiac amyloidosis by $(99 \mathrm{~m})$ Tc-PYP scintigraphy. Hell. J. Nucl. Med. 18, 42-50 (2015).

38. Adams, D., Theaudin, M., Cauquil, C., Algalarrondo, V. \& Slama, M. FAP neuropathy and emerging treatments. Curr. Neurol. Neurosci. Rep. 14, 435 (2014).

39. Kerschen, P. \& Plante-Bordeneuve, V. Current and future treatment approaches in transthyretin familial amyloid polyneuropathy. Curr. Treat. Options Neurol. 18, 53 (2016).

40. Rocha, A. \& Lobato, L. Liver transplantation in transthyretin amyloidosis: Characteristics and management related to kidney disease. Transplant. Rev. (Orlando). 31, 115-120 (2017).

41. Chakraborty, R., Muchtar, E. \& Gertz, M. A. Newer therapies for amyloid cardiomyopathy. Curr. Heart Fail. Rep. 13, 237-246 (2016).

42. Plante-Bordeneuve, V. Transthyretin familial amyloid polyneuropathy: an update. J. Neurol. 265, 976-983 (2018).

43. Rizk, M. \& Tuzmen, S. Update on the clinical utility of an RNA interferencebased treatment: focus on Patisiran. Pharmgenomics Pers. Med. 10, 267-278 (2017).

44. Mankad, A. K. \& Shah, K. B. Transthyretin cardiac amyloidosis. Curr. Cardiol. Rep. 19, 97 (2017).

45. Kumar, S. et al. Revised prognostic staging system for light chain amyloidosis incorporating cardiac biomarkers and serum free light chain measurements. J. Clin. Oncol. 30, 989-995 (2012).

46. Wechalekar, A. D. et al. A European collaborative study of treatment outcomes in 346 patients with cardiac stage III AL amyloidosis. Blood 121, 3420-3427 (2013).

47. Palladini, G. et al. A European collaborative study of cyclophosphamide, bortezomib, and dexamethasone in upfront treatment of systemic AL amyloidosis. Blood 126, 612-615 (2015).

48. Jones, N. F., Hilton, P. J., Tighe, J. R. \& Hobbs, J. R. Treatment of "primary" renal amyloidosis with melphalan. Lancet 2, 616-619 (1972).

49. Comenzo, R. L. et al. Dose-intensive melphalan with blood stem cell support for the treatment of $\mathrm{AL}$ amyloidosis: one-year follow-up in five patients. Blood 88, 2801-2806 (1996).

50. Dhodapkar, M. V. et al. Treatment of AL-amyloidosis with dexamethasone plus alpha interferon. Leuk. Lymphoma. 27, 351-356 (1997).

51. Palladini, G. et al. Association of melphalan and high-dose dexamethasone is effective and well tolerated in patients with AL (primary) amyloidosis who are ineligible for stem cell transplantation. Blood 103, 2936-2938 (2004)

52. Dispenzieri, A. et al. Poor tolerance to high doses of thalidomide in patients with primary systemic amyloidosis. Amyloid 10, 257-261 (2003).

53. Seldin, D. C. et al. Tolerability and efficacy of thalidomide for the treatment of patients with light chain-associated (AL) amyloidosis. Clin. Lymphoma 3, 241-246 (2003).

54. Dispenzieri, A. et al. The activity of lenalidomide with or without dexamethasone in patients with primary systemic amyloidosis. Blood 109 465-470 (2007).
55. Sanchorawala, $\mathrm{V}$. et al. Lenalidomide and dexamethasone in the treatment of AL amyloidosis: results of a phase 2 trial. Blood 109, 492-496 (2007).

56. Sanchorawala, V. et al. Pomalidomide and dexamethasone in the treatment of AL amyloidosis: results of a phase 1 and 2 trial. Blood 128, 1059-1062 (2016).

57. Dispenzieri, A. et al. Activity of pomalidomide in patients with immunoglobulin light-chain amyloidosis. Blood 119, 5397-5404 (2012).

58. Hegenbart, U. et al. Lenalidomide/melphalan/dexamethasone in newly diagnosed patients with immunoglobulin light chain amyloidosis: results of a prospective phase 2 study with long-term follow up. Haematologica 102, 1424-1431 (2017).

59. Tapan, U. et al. Increases in B-type natriuretic peptide (BNP) during treatment with lenalidomide in AL amyloidosis. Blood 116, 5071-5072 (2010).

60. Dispenzieri, A. et al. Discordance between serum cardiac biomarker and immunoglobulin-free light-chain response in patients with immunoglobulin light-chain amyloidosis treated with immune modulatory drugs. Am. J. Hematol. 85, 757-759 (2010).

61. Sher, T. \& Gertz, M. A. Stem cell transplantation for immunoglobulin light chain amyloidosis. Curr. Probl. Cancer 41, 129-137 (2017).

62. Cordes, S. et al. Ten-year survival after autologous stem cell transplantation for immunoglobulin light chain amyloidosis. Cancer 118, 6105-6109 (2012).

63. Gertz, M. A. et al. Stem cell transplantation compared with melphalan plus dexamethasone in the treatment of immunoglobulin light-chain amyloidosis. Cancer 122, 2197-2205 (2016).

64. Gertz, M. A. et al. Refinement in patient selection to reduce treatment-related mortality from autologous stem cell transplantation in amyloidosis. Bone Marrow Transplant. 48, 557-561 (2013).

65. Vaxman, I., Sidiqi, M. H. \& Gertz, M. Venetoclax for the treatment of multiple myeloma. Expert Rev. Hematol. 11, 915-920 (2018).

66. Landau, $\mathrm{H}$. et al. Bortezomib and dexamethasone consolidation following risk-adapted melphalan and stem cell transplantation for patients with newly diagnosed light-chain amyloidosis. Leukemia 27, 823-828 (2013)

67. Huang, $X$. et al. Induction therapy with bortezomib and dexamethasone followed by autologous stem cell transplantation versus autologous stem cell transplantation alone in the treatment of renal $\mathrm{AL}$ amyloidosis: a randomized controlled trial. BMC Med. 12, 2 (2014).

68. Palladini, G. et al. Oral melphalan and dexamethasone grants extended survival with minimal toxicity in $\mathrm{AL}$ amyloidosis: long-term results of a riskadapted approach. Haematologica 99, 743-750 (2014).

69. Wechalekar, A. D. et al. Safety and efficacy of risk-adapted cyclophosphamide, thalidomide, and dexamethasone in systemic AL amyloidosis. Blood 109, 457-464 (2007)

70. Cibeira, M. T. et al. A phase II trial of lenalidomide, dexamethasone and cyclophosphamide for newly diagnosed patients with systemic immunoglobulin light chain amyloidosis. Br. J. Haematol. 170, 804-813 (2015).

71. Kastritis, E. et al. A phase $1 / 2$ study of lenalidomide with low-dose oral cyclophosphamide and low-dose dexamethasone ( $\mathrm{RdC}$ ) in $\mathrm{AL}$ amyloidosis. Blood 119, 5384-5390 (2012).

72. Mikhael, J. R. et al. Cyclophosphamide-bortezomib-dexamethasone (CyBorD) produces rapid and complete hematologic response in patients with $\mathrm{AL}$ amyloidosis. Blood 119, 4391-4394 (2012).

73. Kastritis, E. et al. Bortezomib, melphalan, and dexamethasone for light-chain amyloidosis. J. Clin. Oncol. 38, 3252-3260 (2020). https://doi.org/10.1200/ JCO.20.01285.

74. Manwani, R. et al. A prospective observational study of 915 patients with systemic AL amyloidosis treated with upfront bortezomib. Blood 134, 2271-2280 (2019).

75. Muchtar, E. et al. Interphase fluorescence in situ hybridization in untreated AL amyloidosis has an independent prognostic impact by abnormality type and treatment category. Leukemia 31, 1562-1569 (2017).

76. Kastritis, E. et al. Addition of cyclophosphamide and higher doses of dexamethasone do not improve outcomes of patients with $\mathrm{AL}$ amyloidosis treated with bortezomib. Blood. Cancer J. 7, e570 (2017).

77. Sher, T., Fenton, B., Akhtar, A. \& Gertz, M. A. First report of safety and efficacy of daratumumab in 2 cases of advanced immunoglobulin light chain amyloidosis. Blood 128, 1987-1989 (2016).

78. Kaufman, G. P. et al. Daratumumab yields rapid and deep hematologic responses in patients with heavily pretreated $\mathrm{AL}$ amyloidosis. Blood 130, 900-902 (2017).

79. Abeykoon, J. P. et al. Daratumumab-based therapy in patients with heavilypretreated AL amyloidosis. Leukemia 33, 531-536 (2019). 
80. Khouri, J. et al. Daratumumab proves safe and highly effective in AL amyloidosis. Br. J. Haematol. 185, 342-344 (2019).

81. Cohen, O. C. et al. Rapid response to single agent daratumumab is associated with improved progression-free survival in relapsed/refractory AL amyloidosis. Amyloid 27, 200-205 (2020)

82. Roussel, M. et al. A prospective phase 2 trial of daratumumab in patients with previously treated systemic light-chain amyloidosis. Blood 135, 1531-1540 (2020).

83. Sanchorawala, $V$. et al. Safety, tolerability, and response rates of daratumumab in relapsed $\mathrm{AL}$ amyloidosis: results of a phase 2 study. Blood 135 1541-1547 (2020).

84. Chung, A. et al. Organ responses with daratumumab therapy in previously treated AL amyloidosis. Blood Adv. 4, 458-466 (2020).

85. Kimmich, C. R. et al. Daratumumab for systemic AL amyloidosis: prognostic factors and adverse outcome with nephrotic-range albuminuria. Blood 135 1517-1530 (2020).

86. Palladini, G. et al. Daratumumab plus CyBorD for patients with newly diagnosed AL amyloidosis: safety run-in results of ANDROMEDA. Blood 136 71-80 (2020)

87. Kastritis, E. et al. Subcutaneous daratumumab + cyclophosphamide, bortezomib, and dexamethasone (CYBORD) in patients with newly diagnosed light chain (AL) amyloidosis: primary results from the phase 3 andromeda study. EHA Library 303396, LB2604 (2020).

88. Bochtler, T. et al. Translocation $t(11 ; 14)$ is associated with adverse outcome in patients with newly diagnosed $\mathrm{AL}$ amyloidosis when treated with bortezomib-based regimens. J. Clin. Oncol. 33, 1371-1378 (2015).

89. Leung, N., Thome, S. D. \& Dispenzieri, A. Venetoclax induced a complete response in a patient with mmunoglobulin light chain amyloidosis plateaued oncyclophosphamide, bortezomib and dexamethasone. Haematologica 103, e135-e137 (2018).

90. Ghilardi, G. et al. Venetoclax plus daratumumab induce hematological CR and organ response in an AL amyloidosis patient with $\mathrm{t}(11 ; 14)$. Amyloid 26 173-174 (2019).

91. Gran, C., Borg Bruchfeld, J., Ellin, F. \& Nahi, H. Rapid complete response to single-agent $\mathrm{BCl}-2$ inhibitor venetoclax in a heart-transplanted patient with triple refractory immunoglobulin light-chain amyloidosis. Acta Haematol. 143, 500-503 (2020)

92. Yip, P. L., Lau, J. S. M. \& Lam, C. P. Venetoclax monotherapy induced rapid and sustained response in a frail patient with refractory $\mathrm{AL}$ amyloidosis: less is more? Int. J. Hematol. 112, 234-237 (2020).

93. Sidiqi, M. H. et al. Venetoclax for the treatment of translocation $(11 ; 14) \mathrm{AL}$ amyloidosis. Blood Cancer J. 10, 55 (2020).

94. Cohen, A. D. et al. Safety and efficacy of carfilzomib (CFZ) in previouslytreated systemic light-chain (AL) amyloidosis. Blood 128, 645-645 (2016).

95. Chari, A. et al. Cardiac events in real-world multiple myeloma patients treated with carfilzomib: a retrospective claims database analysis. Blood $\mathbf{1 2 8}$ 3319-3319 (2016).

96. Manwani, R. et al. Carfilzomib is an effective upfront treatment in AL amyloidosis patients with peripheral and autonomic neuropathy. Br. J. Haematol. 187, 638-641 (2019).

97. Sanchorawala, V. et al. A phase $1 / 2$ study of the oral proteasome inhibitor ixazomib in relapsed or refractory AL amyloidosis. Blood 130, 597-605 (2017).

98. Cohen, O. C. et al. Use of ixazomib, lenalidomide and dexamethasone in patients with relapsed amyloid light-chain amyloidosis. Br. J. Haemato/ 189 643-649 (2020).

99. Lentzsch, S. et al. Bendamustine with dexamethasone in relapsed/refractory systemic light-chain amyloidosis: results of a phase II study. J. Clin. Oncol. $\mathbf{3 8}$ 1455-1462 (2020).
100. Wechalekar, A. D. \& Whelan, C. Encouraging impact of doxycycline on early mortality in cardiac light chain (AL) amyloidosis. Blood Cancer J. 7, e546 (2017)

101. Cardoso, I., Martins, D., Ribeiro, T., Merlini, G. \& Saraiva, M. J. Synergy of combined doxycycline/TUDCA treatment in lowering Transthyretin deposition and associated biomarkers: studies in FAP mouse models. J. Transl. Med. 8, 74 (2010).

102. Cardoso, I. \& Saraiva, M. J. Doxycycline disrupts transthyretin amyloid: evidence from studies in a FAP transgenic mice model. FASEB J. 20, 234-239 (2006).

103. Ward, J. E. et al. Doxycycline reduces fibril formation in a transgenic mouse model of AL amyloidosis. Blood 118, 6610-6617 (2011).

104. Obici, L. et al. Doxycycline plus tauroursodeoxycholic acid for transthyretin amyloidosis: a phase II study. Amyloid 19, 34-36 (2012).

105. Kumar, S. K. et al. Doxycycline used as post transplant antibacterial prophylaxis improves survival in patients with light chain amyloidosis undergoing autologous stem cell transplantation. Blood 120, 3138-3138 (2012).

106. D'Souza, A. et al. Adjuvant doxycycline to enhance anti-amyloid effects: results from the dual phase 2 trial. EClinicalMedicine. 23, 100361 (2020).

107. Valero-Muñoz, M. et al. Doxycycline decreases amyloidogenic light chaininduced autophagy in isolated primary cardiac myocytes. Int. J. Cardiol. 321 133-136 (2020).

108. Solomon, A., Weiss, D. T. \& Wall, J. S. Immunotherapy in systemic primary (AL) amyloidosis using amyloid-reactive monoclonal antibodies. Cancer Biother Radiopharm. 18, 853-860 (2003).

109. Edwards, C. V. et al. One year follow up analysis of the phase $1 \mathrm{a} / \mathrm{b}$ study of chimeric fibril-reactive monoclonal antibody 11-1F4 in patients with AL amyloidosis. Amyloid 26, 115-116 (2019).

110. Wall, J. S., Foster, J. S., Martin, E. B. \& Kennel, S. J. Pretargeting immunotherapy: a novel treatment approach for systemic amyloidosis. Pharm. Pat. Anal. $\mathbf{6}$ 215-223 (2017).

111. Edwards, C. V. et al. Interim analysis of the phase $1 \mathrm{a} / \mathrm{b}$ study of chimeric fibrilreactive monoclonal antibody $11-1 \mathrm{~F} 4$ in patients with $\mathrm{AL}$ amyloidosis. Amyloid 24, 58-59 (2017).

112. Leung, $\mathrm{N}$. et al. The impact of dialysis on the survival of patients with immunoglobulin light chain (AL) amyloidosis undergoing autologous stem cell transplantation. Nephrol. Dial. Transplant. 31, 1284-1289 (2016).

113. Batalini, F. et al. High-dose melphalan and stem cell transplantation in patients on dialysis due to immunoglobulin light-chain amyloidosis and monoclonal immunoglobulin deposition disease. Biol. Blood Marrow Transplant. 24, 127-132 (2018).

114. Scully, M. S. et al. Total artificial heart implantation as a bridge to heart transplantation in an active duty service member with amyloid cardiomyopathy. Mil. Med. 182, e1858-e1860 (2017).

115. Kristen, A. V. et al. Improved outcomes after heart transplantation for cardiac amyloidosis in the modern era. J. Heart Lung Transplant. 37, 611-618 (2018).

116. Huh, J. Y. et al. Sequential heart and autologous stem cell transplantation for light-chain cardiac amyloidosis. Blood Res. 52, 221-224 (2017).

117. Sousa, M., Monohan, G., Rajagopalan, N., Grigorian, A. \& Guglin, M. Heart transplantation in cardiac amyloidosis. Heart Fail. Rev. 22, 317-327 (2017)

118. Grogan, M. et al. Long term outcomes of cardiac transplant for immunoglobulin light chain amyloidosis: the Mayo Clinic experience. World J. Transplant. 6, 380-388 (2016)

119. Meyers, D. E. et al. Fatal cardiac and renal allograft rejection with lenalidomide therapy for light-chain amyloidosis. Am. J. Transplant. 13, 2730-2733 (2013). 